\title{
Ultrasensitive Displacement Noise Measurement of Carbon Nanotube Mechanical Resonators
}

\author{
S. L. de Bonis, ${ }^{\dagger}, \S$ C. Urgell, ${ }^{\dagger, \S}$ W. Yang, ${ }^{\dagger}$ C. Samanta, ${ }^{\dagger}$ A. Noury, ${ }^{\dagger, \|}$ J. Vergara-Cruz, ${ }^{\dagger}$ Q. Dong, ${ }^{\dagger}$ Y. Jin, ${ }^{\dagger}$ \\ and A. Bachtold* ${ }^{*} \dagger$ \\ ${ }^{\dagger}$ ICFO - Institut de Ciencies Fotoniques, The Barcelona Institute of Science and Technology, 08860 Castelldefels, Barcelona, Spain \\ ${ }^{\ddagger}$ Centre de Nanosciences et de Nanotechnologies, CNRS, University of Paris-Sud, University of Paris-Saclay, C2N Marcoussis, \\ 91460 Marcoussis, France
}

Supporting Information

ABSTRACT: Mechanical resonators based on a single carbon nanotube are exceptional sensors of mass and force. The force sensitivity in these ultralight resonators is often limited by the noise in the detection of the vibrations. Here, we report on an ultrasensitive scheme based on a RLC resonator and a low-temperature amplifier to detect nanotube vibrations. We also show a new fabrication process of
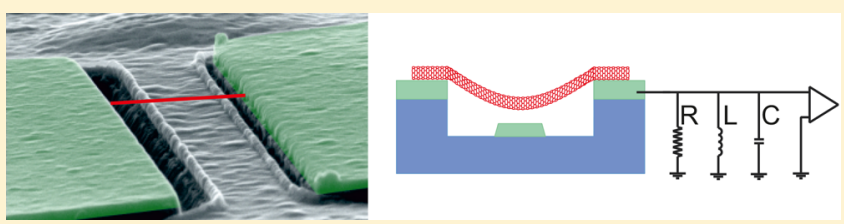
electromechanical nanotube resonators to reduce the separation between the suspended nanotube and the gate electrode down to $\sim 150 \mathrm{~nm}$. These advances in detection and fabrication allow us to reach $0.5 \mathrm{pm} / \sqrt{\mathrm{Hz}}$ displacement sensitivity. Thermal vibrations cooled cryogenically at $300 \mathrm{mK}$ are detected with a signal-to-noise ratio as high as $17 \mathrm{~dB}$. We demonstrate $4.3 \mathrm{zN} / \sqrt{\mathrm{Hz}}$ force sensitivity, which is the best force sensitivity achieved thus far with a mechanical resonator. Our work is an important step toward imaging individual nuclear spins and studying the coupling between mechanical vibrations and electrons in different quantum electron transport regimes.

KEYWORDS: Nanomechanical resonators, displacement sensitivity, force sensitivity, NEMS, carbon nanotube

$\mathrm{T}^{\mathrm{k}}$ he smallest operational mechanical resonators are based on low-dimensional materials, such as carbon nanotubes, ${ }^{1}$ graphene, ${ }^{2-4}$ semiconducting nanowires, ${ }^{5-8}$ and levitated particles. ${ }^{9,10}$ Such resonators are fantastic sensors of external forces $^{11-14}$ and the adsorption of mass. ${ }^{15-17}$ They also provide a versatile platform for fundamental science, including the study of noise, ${ }^{18-20}$ nonlinear phenomena, ${ }^{21-24}$ electronphonon coupling, ${ }^{25-29}$ and light-matter interaction. ${ }^{30,31}$ The greatest challenge with these tiny resonators is to transduce their mechanical vibrations into a measurable electrical or optical output signal. Novel detection methods have been continuously developed over the years. ${ }^{32-47}$ This effort has often been paid off with the improvement of sensing capabilities and the measurement of unexpected phenomena.

Care has to be taken to avoid heating when improving the detection of the motion. The transduction of the motion is achieved by applying some input power to the resonator. In the case of nanotube resonators, the input power is usually related to the oscillating voltage applied across the nanotube ${ }^{1}$ or the laser beam illuminating the nanotube. ${ }^{38,47}$ The displacement sensitivity becomes better when increasing the input power. However, the input power has to be kept low enough to avoid electrical Joule heating and optical adsorption heating. Heating is especially prominent in tiny objects, such as nanotubes, because of their small heat capacity. Heating is detrimental, because it deteriorates the force and the mass sensitivity and increases the number of quanta of vibrational energy.
Here, we report on a novel detection method that allows us to measure the mechanical vibrations of nanotube resonators with an unprecedented sensitivity. The detection consists in measuring the electrical signal with a RLC resonator and a high electron mobility transistor (HEMT) amplifier cooled at liquid-helium temperature. In order to further improve the detection, we developed a new fabrication process to enhance the capacitive coupling between the ultraclean carbon nanotube and the gate electrode. This allows us to achieve $1.7 \mathrm{pm} / \sqrt{\mathrm{Hz}}$ displacement sensitivity when the temperature of the measured eigenmode is $120 \mathrm{mK}$. At higher temperature, the resonator can be probed with larger input power, so that the sensitivity reaches $0.5 \mathrm{pm} / \sqrt{\mathrm{Hz}}$ at $300 \mathrm{mK}$.

We use a new fabrication process to grow ultraclean carbon nanotube resonators suspended over shallow trenches. Figure 1a shows an $\sim 1.3 \mu \mathrm{m}$ long nanotube contacted electrically to two electrodes and separated from the local gate electrode by $\sim 150 \mathrm{~nm}$. The electrodes made from platinum with a tungsten adhesion layer are evaporated on top of silicon dioxide grown by plasma-enhanced chemical vapor deposition. Nanotubes are grown by the "fast heating" chemical vapor deposition method in the last fabrication step. ${ }^{48}$ This method consists in rapidly sliding the quartz tube through the oven under a flow of

Received: June 15, 2018

Revised: July 16, 2018

Published: July 31, 2018 

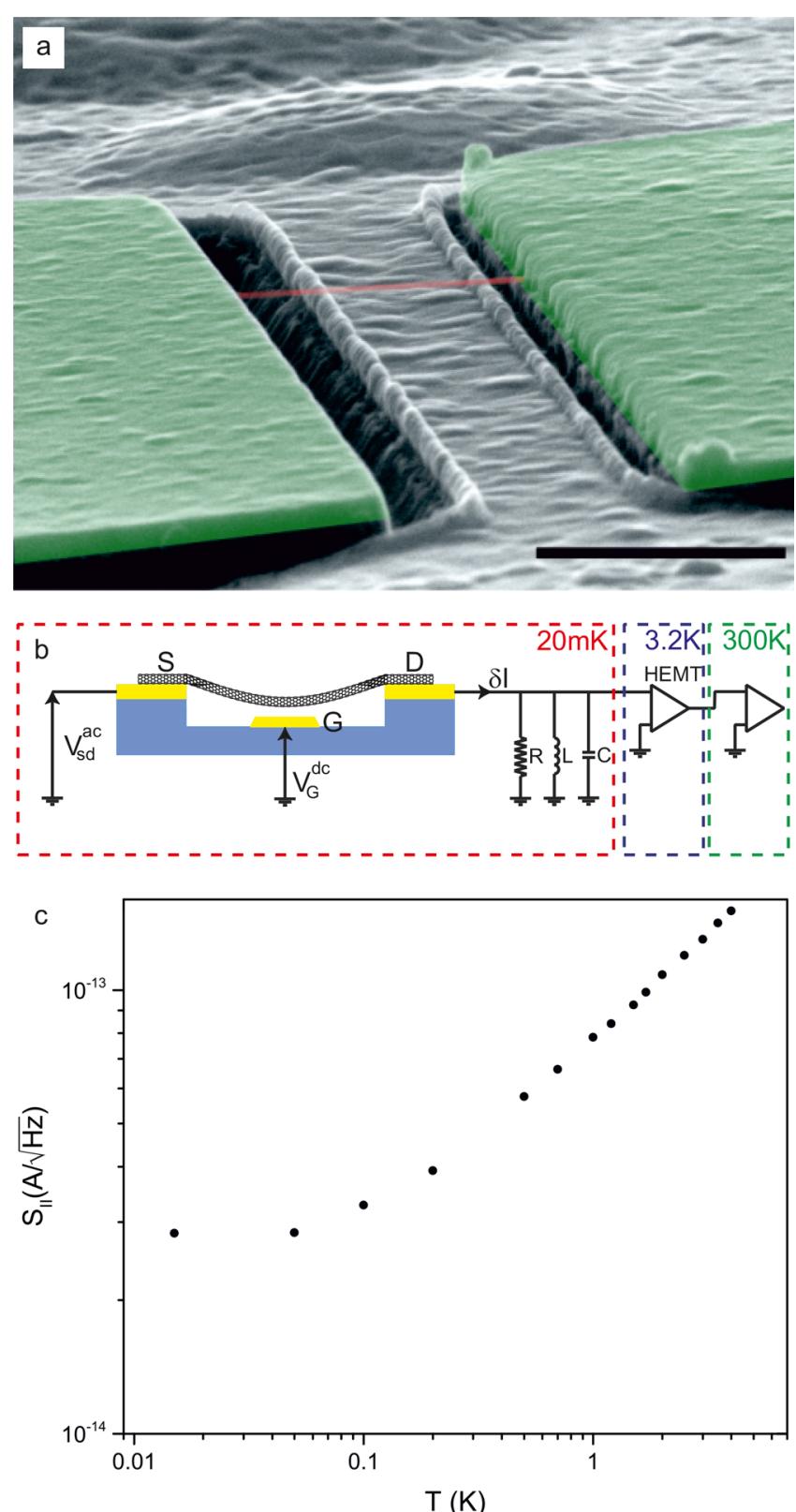

Figure 1. Nantotube resonator and electrical circuit for the detection of the vibrations. (a) False-color scanning electron microscopy image of a typical nanotube resonator fabricated with the "fast heating" chemical vapor deposition method. The $\sim 20 \mathrm{~nm}$ high ridges at the edges of the gate electrodes are attributed to resist residues. The scale bar is $1 \mu \mathrm{m}$. (b) Schematic of the measurement of the nanotube vibrations using the RLC resonator and the HEMT amplifier cooled at $3.2 \mathrm{~K}$. The base temperature of the cryostat is $\sim 20 \mathrm{mK}$. An oscillating voltage with amplitude $V_{s d}^{\text {ac }}$ is applied between electrodes $S$ and $D$, and a constant voltage $V_{\mathrm{G}}^{\mathrm{dc}}$ is applied to electrode $G$. (c) Temperature dependence of the current noise floor of the circuit measured at $\omega_{\text {RLC }}$.

methane, so that the sample moves from a position outside of the oven to the center of the oven, whose temperature is $T_{\text {growth }}$ $=820{ }^{\circ} \mathrm{C}$. This growth process has two assets compared to the usual growth of nanotube resonators. ${ }^{37}$ It allows us to suspend nanotubes over wide trenches. In addition, the electrodes are less prone to melt and change shape.

Mechanical vibrations are detected electrically using a RLC resonator and a HEMT amplifier cooled at liquid-helium temperature (Figure 1b). Displacement modulation is transduced capacitively into current modulation by applying an input oscillating voltage $V_{\mathrm{sd}}^{\mathrm{ac}}$ across the nanotube. ${ }^{1,12,37}$ The frequency $\omega_{\text {sd }} / 2 \pi$ of the oscillating voltage is set to match $\omega_{\text {sd }}$ $=\omega_{0} \pm \omega_{\mathrm{RLC}}$, where $\omega_{0} / 2 \pi$ is the resonance frequency of the nanotube resonator and $\omega_{\mathrm{RLC}} / 2 \pi=1.25 \mathrm{MHz}$ the resonance frequency of the RLC resonator. Driven vibrations are measured with the two-source method. ${ }^{1}$ Thermal vibrations are measured by recording the current noise at $\sim \omega_{\mathrm{RLC}}{ }^{12,37}$ These current noise measurements are similar to those recently carried out on quantum electron devices. ${ }^{49-51}$

The RLC resonator and the HEMT amplifier ${ }^{52}$ allow us to reduce the current noise floor at $\sim \omega_{\mathrm{RLC}}$ down to $28 \mathrm{fA} / \sqrt{\mathrm{Hz}}$ below $\sim 100 \mathrm{mK}$ (Figure 1c). The current noise floor is temperature dependent above $\sim 100 \mathrm{mK}$ because of the Johnson-Nyquist noise of the impedance of the RLC resonator. Below $\sim 100 \mathrm{mK}$, the Johnson-Nyquist noise becomes vanishingly small. The noise floor is then given by the current noise $(6.2 \mathrm{fA} / \sqrt{\mathrm{Hz}})$ and the voltage noise $(0.16 \mathrm{nV} / \sqrt{\mathrm{Hz}})$ of the HEMT amplifier and the voltage noise of the room-temperature amplifier. The gain of the HEMT amplifier is set at 5.6. The inductance of the circuit is given by the $66 \mu \mathrm{H}$ inductance soldered onto a printed-circuit board (PCB). The $242 \mathrm{pF}$ capacitance measured from the RLC resonance frequency comes from the capacitance of the radio frequency cables and the low-pass filter VLFX-80 between the device and the HEMT. The $7.52 \mathrm{k} \Omega$ resistance obtained from the $87 \mathrm{kHz}$ line width of the RLC resonator is attributed to the $10 \mathrm{k} \Omega$ resistance soldered onto the PCB and the input impedance of the HEMT amplifier.

The lowest-lying flexural eigenmodes are identified by capacitively driving the resonator with an oscillating force and measuring the motion with the two-source method. ${ }^{1}$ The dependence of the resonance frequency as a function of the static voltage $V_{\mathrm{G}}^{\mathrm{dc}}$ applied to the gate electrode demonstrates that the measured resonance is related to a mechanical eigenmode of the nanotube (Figure 2a). The amplitude of the lowest-frequency resonance is much larger than that of the second detected resonance (Figure $2 \mathrm{~b}$ ). We conclude that the detected eignmodes are polarized in the direction perpendicular to the surface of the gate electrode to a good approximation and that eigenmodes polarized in the parallel direction cannot be detected.

The thermal vibrations are recorded at the base temperature of the dilution cryostat (Figure 2c). We switch off the driving force, and the displacement noise is recorded with the method described in refs. ${ }^{12,37}$ The quality factor is $Q=627,000$ with the gate voltage set at $V_{\mathrm{G}}^{\mathrm{dc}}=-0.21 \mathrm{~V}$. We choose this gate voltage so that the electron transport is not in the Coulomb blockade regime. The $Q$-factor becomes lower at more negative gate voltages because of electrical losses ${ }^{53}$ and at positive gate voltages due to Coulomb blockade. ${ }^{25} \mathrm{We}$ measure the dependence of the variance of the displacement $\left\langle\delta z^{2}\right\rangle$ on the cryostat temperature $T$ (Figure $2 \mathrm{~d}$ ). The linear dependence is in agreement with the equipartition theorem $m \omega_{0}^{2}\left\langle\delta z^{2}\right\rangle=$ $k_{\mathrm{b}} T$, where $m$ is the effective mass of the resonator. We obtain $m=8.6$ ag from the slope, which is consistent with the mass expected for an $\sim 1.3 \mu \mathrm{m}$ long nanotube. Below $\sim 120 \mathrm{mK}$, the eigenmode does not thermalize well with the cryostat (Figure $2 \mathrm{~d})$. Measurements on a second nanotube resonator show that the eigenmode reaches $\sim 50 \mathrm{mK}$ (Supporting Information). The origin of this poor thermalization at low temperature may 

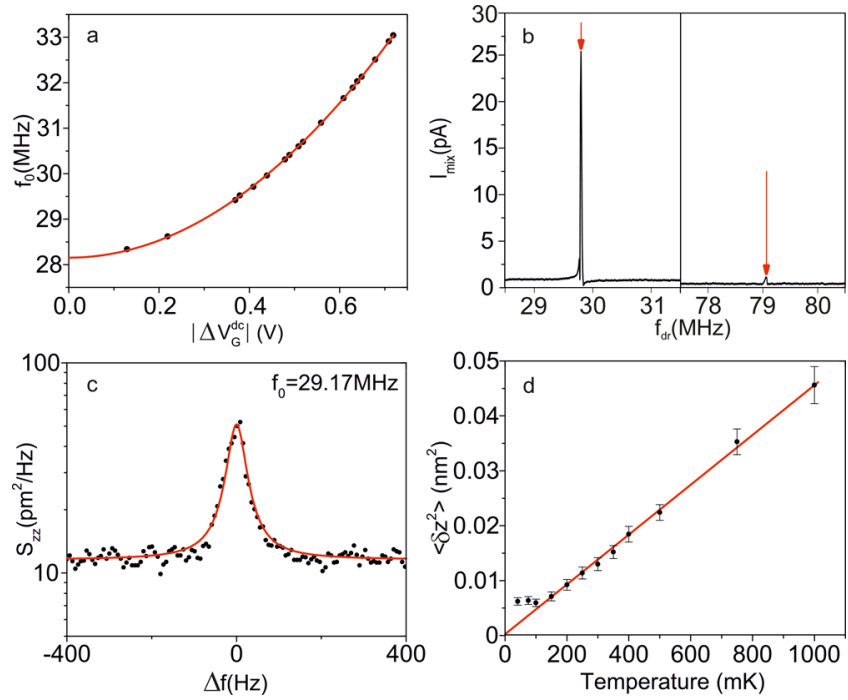

Figure 2. Driven and thermal vibrations of the nanotube resonator. (a) Gate voltage dependence of the resonance frequency of the fundamental eigenmode. The small positive offset voltage $V_{\text {off }}=0.119$ $\mathrm{V}$ due to the work function difference between the nanotube and the gate electrode is subtracted from the applied $V_{\mathrm{G}}^{\mathrm{dc}}$ value. (b) Driven response of the two lowest-frequency detected mechanical eigenmodes as a function of the drive frequency measured with the twosource method. The resonances are indicated by two red arrows. (c) Spectrum of the displacement noise of the fundamental eigenmode measured at the base temperature of the cryostat when applying $V_{\mathrm{G}}^{\mathrm{dc}}=$ $-0.21 \mathrm{~V}$ and $V_{\text {sd }}^{\mathrm{ac}}=40 \mu \mathrm{V}$. The resonance frequency $f_{0}$ is given in the figure. (d) Variance of the displacement measured as a function of cryostat temperature.

be related to a nonthermal force noise, such as the electrostatic force noise related to the voltage noise in the device. ${ }^{54}$

The force sensitivity derived from the noise spectrum in Figure $2 \mathrm{c}$ is $\sqrt{S_{\mathrm{FF}}}=4.3 \pm 2.9 \mathrm{zN} / \sqrt{\mathrm{Hz}}$. Because the thermal resonance is described by a Lorentzian line shape, the force sensitivity is quantified from the total displacement noise at resonance frequency using $S_{\mathrm{FF}}=S_{z z}\left(\omega_{0}\right) /\left|\chi\left(\omega_{0}\right)\right|^{2}$ with the mechanical susceptibility $\left|\chi\left(\omega_{0}\right)\right|=Q / m \omega_{0}^{2}$. The force sensitivity is given to a large extent by the thermal force noise of the resonator $\sqrt{S_{\mathrm{FF}}^{\text {th }}}=\sqrt{4 k_{\mathrm{b}} T m \omega_{0} / Q}=4.0 \mathrm{zN} / \sqrt{\mathrm{Hz}}$, which is the fundamental limit of the force sensitivity set by the fluctuation-dissipation theorem. The noise of the imprecision in the detection contributes to the force sensitivity by a low amount. The error bar in the estimation of the force sensitivity originates essentially from the uncertainty in the nanotube diameter and the separation between the nanotube and the gate electrode. Table 1 shows that the force sensitivity measured in this work is better than what is reported with resonators microfabricated from bulk material ${ }^{55-57}$ and resonators based on nanotube, ${ }^{12}$ semiconducting nanowire, ${ }^{13,14,58}$ graphene, ${ }^{54}$ and levitating particles. ${ }^{18}$ In ref 37 , the reported thermal force noise is lower but the crosscorrelation noise measurement does not quantify the force noise due to the imprecision in the detection so that the total force noise cannot be quantified.

We now look at how the displacement noise is affected by the input power related to the oscillating voltage $V_{\mathrm{sd}}^{\text {ac }}$ (Figures $3 \mathrm{a}-\mathrm{d})$. The variance of the displacement increases abruptly above $V_{\mathrm{sd}}^{\mathrm{ac}} \simeq 80 \mu \mathrm{V}$ when the cryostat is at base temperature (Figure $3 \mathrm{c}$ ). This indicates the rise of the thermal vibration amplitude due to Joule heating. By contrast, the variance of the displacement remains constant over the whole range of $V_{\text {sd }}^{\mathrm{ac}}$ that we apply when the cryostat temperature is set at $300 \mathrm{mK}$ (Figure 3d).

Our detection scheme allows us to reach an excellent displacement sensitivity for input powers below the onset of Joule heating. The displacement sensitivity is given by the noise floor of the spectrum of thermal vibrations. The displacement sensitivity gets better when increasing $V_{\text {sd }}^{\text {ac }}$ (Figures 3e,f). The displacement sensitivity at base temperature is $1.7 \mathrm{pm} / \sqrt{\mathrm{Hz}}$ at $V_{\mathrm{sd}}^{\mathrm{ac}}=80 \mu \mathrm{V}$ before that Joule heating starts to increase the variance of the displacement. When the cryostat temperature is set at $300 \mathrm{mK}$, the displacement sensitivity is $0.5 \mathrm{pm} / \sqrt{\mathrm{Hz}}$ at the largest $V_{\mathrm{sd}}^{\mathrm{ac}}$ value that we apply. The corresponding signal-to-noise ratio in the spectrum of thermal vibrations is $17 \mathrm{~dB}$ (Figure $3 \mathrm{~b}$ ). The measured displacement sensitivity $S_{\mathrm{zz}}^{\mathrm{imp}}$ scales as $\left(1 / V_{\mathrm{sd}}^{\mathrm{ac}}\right)^{2}$ (Figures $3 \mathrm{e}, \mathrm{f}$ ), indicating that $S_{\mathrm{zz}}^{\mathrm{imp}}$ is limited by the noise of the detection circuit and not by the electron shot noise through the nanotube. ${ }^{59}$ The $Q$-factor in Figure 3 is lower than that in Figure $2 \mathrm{c}$ due to an unknown reason while cycling the cryostat through room temperature; the resonance frequency and the mass are not modified by the thermal cycling.

In conclusion, we report on a detection scheme of nanotube resonators with an unprecedented displacement sensitivity. It allows us to reach $4.3 \mathrm{zN} / \sqrt{\mathrm{Hz}}$ force sensitivity, which surpasses what has been achieved with mechanical resonators to date. This high force sensitivity is an important step toward

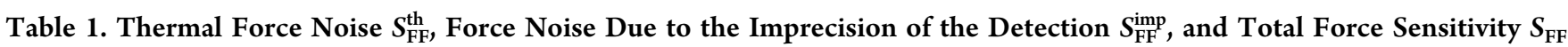
for Different Resonators ${ }^{a}$

$\begin{array}{cl}\sqrt{S_{\mathrm{FF}}^{\text {th }}}(\mathrm{N} / \sqrt{\mathrm{Hz}}) & \sqrt{S_{\mathrm{FF}}^{\text {imp }}}(\mathrm{N} / \sqrt{\mathrm{Hz}}) \\ 4.0 \times 10^{-21} & 1.6 \times 10^{-21} \\ 2.0 \times 10^{-20} & \text { negligible } \\ 2.7 \times 10^{-19} & 2.7 \times 10^{-19} \\ 1.0 \times 10^{-18} & \text { negligible } \\ 5.0 \times 10^{-18} & \text { negligible } \\ 1.6 \times 10^{-19} & 1.0 \times 10^{-19} \\ 5.1 \times 10^{-19} & \text { negligible } \\ 2.0 \times 10^{-17} & \text { negligible } \\ 1.2 \times 10^{-20} & \text { unknown } \\ \sim 1 \times 10^{-21} & \text { unknown }\end{array}$

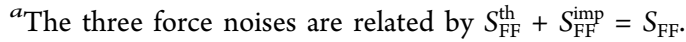

$$
\begin{aligned}
& \sqrt{S_{\mathrm{FF}}}(\mathrm{N} / \sqrt{\mathrm{Hz}}) \\
& 4.3 \times 10^{-21} \\
& 2.0 \times 10^{-20} \\
& 3.9 \times 10^{-19} \\
& 1.0 \times 10^{-18} \\
& 5.0 \times 10^{-18} \\
& 1.9 \times 10^{-19} \\
& 5.1 \times 10^{-19} \\
& 2.0 \times 10^{-17} \\
& \text { unknown } \\
& \text { unknown }
\end{aligned}
$$

description

nanotube (this work)

levitating particle ${ }^{18}$

graphene ${ }^{54}$

silicon nanowire ${ }^{58}$

GaAs/AlGaAs nanowire ${ }^{14}$

microfabricated ladder ${ }^{56}$

microfabricated beam ${ }^{55}$

microfabricated trampoline $\mathrm{e}^{57}$

nanotube ${ }^{12}$

nanotube $^{37}$ 

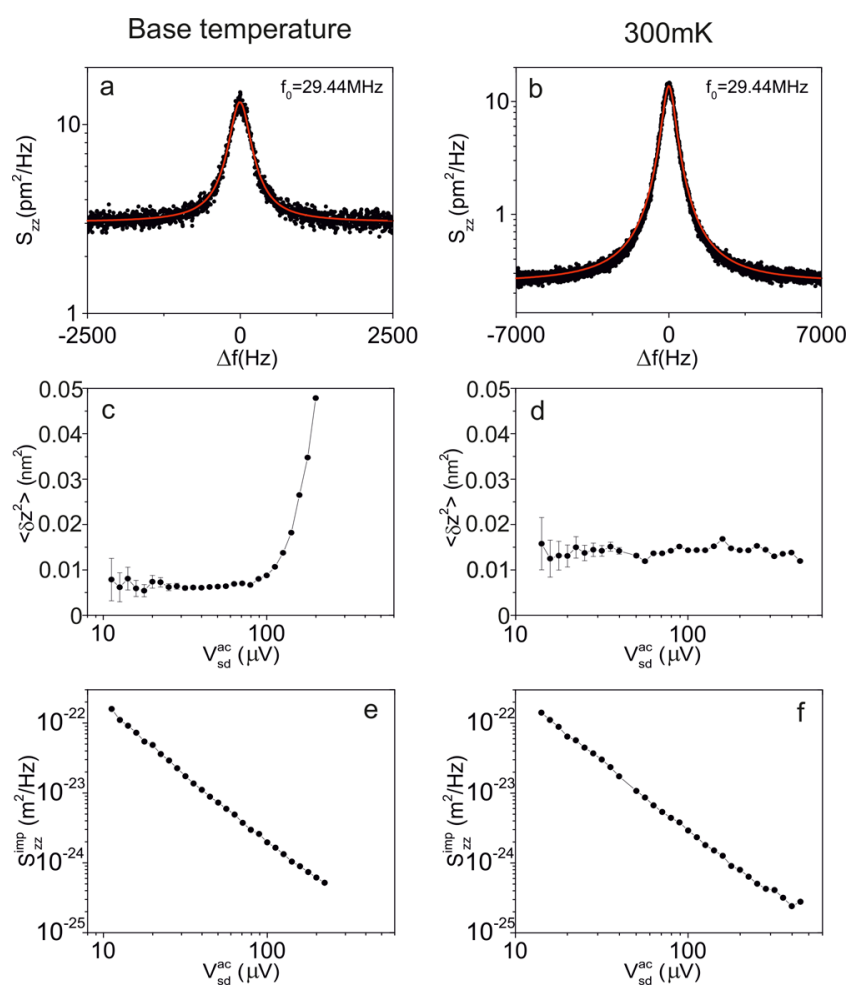

Figure 3. Spectrum of the displacement noise modified by the oscillating voltage with amplitude $V_{\text {sd }}^{\text {ac }}$ applied across the nanotube. (a) Spectrum of the displacement noise of the fundamental eigenmode measured at the base temperature of the cryostat when applying $V_{\mathrm{G}}^{\mathrm{dc}}=$ $-0.255 \mathrm{~V}$ and $V_{\text {sd }}^{\mathrm{ac}}=70 \mu \mathrm{V}$. (b) Same as (a) but with the cryostat temperature set at $300 \mathrm{mK}$ and $V_{\mathrm{sd}}^{\mathrm{ac}}=400 \mu \mathrm{V}$. (c,d) Dependence of the variance of the displacement on $V_{\mathrm{sd}}^{\mathrm{sc}}$ measured at the base temperature of the cryostat and $300 \mathrm{mK}$. (e,f) Dependence of the displacement sensitivity on $V_{\mathrm{sd}}^{\mathrm{ac}}$ measured at the base temperature of the cryostat and $300 \mathrm{mK}$.

detecting individual nuclear spins with nuclear magnetic resonance measurements. ${ }^{60,61}$ The coupling between mechanical vibrations and spins can be achieved by applying a gradient of magnetic field that is generated with the current biased through the gate electrode in Figure $1 a^{58}$ In this context, the new fabrication process of nanotube resonators presented in this Letter is useful for increasing the gradient of the magnetic field, because it reduces the separation between the currentcarrying electrode and the nanotube down to $\sim 150 \mathrm{~nm}$. The device layout might also allow us to carry out magnetic resonance force microscopy (MRFM) measurements to image the location of individual nuclear spins adsorbed along the nanotube. Imaging can be done by periodically applying radio frequency pulses though the current-carrying electrode. ${ }^{62}$ Moreover, the advances in fabrication and detection described in this work offer new possibilities for studying the strong coupling between electrons and vibrations in nanoscale resonators. ${ }^{63-66}$ In the Coulomb blockade regime, the system has been predicted to feature a transition toward a mechanically bistable and blocked-current state. ${ }^{66-69}$ This hitherto unobserved transition is expected to occur at higher temperature for shorter separation between the nanotube and the gate electrode. ${ }^{66}$ The high quality displacement noise spectra reported here might allow us to study this transition in details $^{66}$ as well as the different cooling schemes that have been proposed theoretically using different quantum electron transport regimes. ${ }^{70-74}$

\section{ASSOCIATED CONTENT}

\section{Supporting Information}

The Supporting Information is available free of charge on the ACS Publications website at DOI: 10.1021/acs.nanolett.8b02437.

Further information on the calibration of the displacement sensitivity and the force sensitivity; mechanical and electrical characterization of the device; displacement variance as a function of temperature of a second device (PDF)

\section{AUTHOR INFORMATION}

\section{ORCID}

A. Bachtold: 0000-0002-6145-2479

Present Address

"(A.N.) Laboratoire Charles Coulomb (L2C), Univ Montpellier, CNRS, Montpellier, France.

\section{Author Contributions}

${ }^{\S}$ S.L.d.B. and C.U. contributed equally to this work.

\section{Notes}

The authors declare no competing financial interest.

\section{ACKNOWLEDGMENTS}

This work is supported by the ERC advanced Grant 692876, the Foundation Cellex, the CERCA Programme, AGAUR, Severo Ochoa (SEV-2015-0522), the Grant FIS2015-69831-P of MINECO, and the Fondo Europeo de Desarrollo Regional (FEDER).

\section{REFERENCES}

(1) Sazonova, V.; Yaish, Y.; Ustunel, H.; Roundy, D.; Arias, T. A.; McEuen, P. L. Nature 2004, 431, 284.

(2) Bunch, J. S.; van der Zande, A. M.; Verbridge, S. S.; Frank, I. W.; Tanenbaum, D. M.; Parpia, J. M.; Craighead, H. G.; McEuen, P. L. Science 2007, 315, 490.

(3) Singh, V.; Sengupta, S.; Solanki, H. S.; Dhall, R.; Allain, A.; Dhara, S.; Pant, P.; Deshmukh, M. M. Nanotechnology 2010, 21, 165204.

(4) Will, M.; Hamer, M.; Muller, M.; Noury, A.; Weber, P.; Bachtold, A.; Gorbachev, R. V.; Stampfer, C.; Guttinger, J. Nano Lett. 2017, 17, 5950.

(5) Feng, X. L.; He, R.; Yang, P.; Roukes, M. L. Nano Lett. 2007, 7, 1953.

(6) Gil-Santos, E.; Ramos, D.; Martinez, J.; Fernandez-Regulez, M.; Garcia, R.; San Paulo, A.; Calleja, M.; Tamayo, J. Nat. Nanotechnol. 2010, 5, 641 .

(7) Sansa, M.; Fernandez-Regulez, M.; San Paulo, A.; Perez-Murano, F. Appl. Phys. Lett. 2012, 101, 243115.

(8) Montinaro, M.; Wust, G.; Munsch, M.; Fontana, Y.; RussoAverchi, E.; Heiss, M.; Fontcuberta i Morral, A.; Warburton, R. J.; Poggio, M. Nano Lett. 2014, 14, 4454.

(9) Gieseler, J.; Deutsch, B.; Quidant, R.; Novotny, L. Phys. Rev. Lett. 2012, 109, 103603.

(10) Kiesel, N.; Blaser, F.; Delic, U.; Grass, D.; Kaltenbaek, R.; Aspelmeyer, M. Proc. Natl. Acad. Sci. U. S. A. 2013, 110, 14180.

(11) Nichol, J. M.; Hemesath, E. R.; Lauhon, L. J.; Budakian, R. Appl. Phys. Lett. 2008, 93, 193110.

(12) Moser, J.; Güttinger, J.; Eichler, A.; Esplandiu, M. J.; Liu, D. E.; Dykman, M. I.; Bachtold, A. Nat. Nanotechnol. 2013, 8, 493.

(13) de Lepinay, L. M.; Pigeau, B.; Besga, B.; Vincent, P.; Poncharal, P.; Arcizet, O. Nat. Nanotechnol. 2016, 12, 156. 
(14) Rossi, N.; Braakman, F. R.; Cadeddu, D.; Vasyukov, D.; Tutuncuoglu, G.; Fontcuberta i Morral, A.; Poggio, M. Nat. Nanotechnol. 2016, 12, 150.

(15) Chiu, H.-Y.; Hung, P.; Postma, H. W. C.; Bockrath, M. Nano Lett. 2008, 8, 4342 .

(16) Wang, Z.; Wei, J.; Morse, P.; Dash, J. G.; Vilches, O. E.; Cobden, D. H. Science 2010, 327, 552.

(17) Chaste, J.; Eichler, A.; Moser, J.; Ceballos, G.; Rurali, R.; Bachtold, A. Nat. Nanotechnol. 2012, 7, 301.

(18) Gieseler, J.; Novotny, L.; Quidant, R. Nat. Phys. 2013, 9, 806.

(19) Zhang, Y.; Moser, J.; Güttinger, J.; Bachtold, A.; Dykman, M. I. Phys. Rev. Lett. 2014, 113, 255502.

(20) Miao, T. F.; Yeom, S.; Wang, P.; Standley, B.; Bockrath, M. Nano Lett. 2014, 14, 2982.

(21) Eichler, A.; Moser, J.; Chaste, J.; Zdrojek, M.; Wilson-Rae, I.; Bachtold, A. Nat. Nanotechnol. 2011, 6, 339.

(22) Deng, G.-W.; Zhu, D.; Wang, X.-H.; Zou, C.-L.; Wang, J.-T.; Li, H.-O.; Cao, G.; Liu, D.; Li, Y.; Xiao, M.; Guo, G.-C.; Jiang, K.-L.; Dai, X.-C.; Guo, G.-P. Nano Lett. 2016, 16, 5456.

(23) De Alba, R.; Massel, F.; Storch, I. R.; Abhilash, T. S.; Hui, A.; McEuen, P. L.; Craighead, H. G.; Parpia, J. M. Nat. Nanotechnol. 2016, 11, 741.

(24) Mathew, J. P.; Patel, R. N.; Borah, A.; Vijay, R.; Deshmukh, M. M. Nat. Nanotechnol. 2016, 11, 747.

(25) Lassagne, B.; Tarakanov, Y.; Kinaret, J.; Garcia-Sanchez, D.; Bachtold, A. Science 2009, 325, 1107.

(26) Steele, G. A.; Huttel, A. K.; Witkamp, B.; Poot, M.; Meerwaldt, H. B.; Kouwenhoven, L. P.; van der Zant, H. S. J. Science 2009, 325, 1103.

(27) Ganzhorn, M.; Wernsdorfer, W. Phys. Rev. Lett. 2012, 108, 175502.

(28) Benyamini, A.; Hamo, A.; Kusminskiy, S. V.; von Oppen, F.; Ilani, S. Nat. Phys. 2014, 10, 151.

(29) Ares, N.; Pei, T.; Mavalankar, A.; Mergenthaler, M.; Warner, J. H.; Briggs, G. A. D.; Laird, E. A. Phys. Rev. Lett. 2016, 117, 170801.

(30) Gloppe, A.; Verlot, P.; Dupont-Ferrier, E.; Siria, A.; Poncharal, P.; Bachelier, G.; Vincent, P.; Arcizet, O. Nat. Nanotechnol. 2014, 9, 920.

(31) Reserbat-Plantey, A.; Schadler, K. G.; Gaudreau, L.; Navickaite, G.; Guttinger, J.; Chang, D.; Toninelli, C.; Bachtold, A.; Koppens, F. H. L. Nat. Commun. 2016, 7, 10218.

(32) Purcell, S. T.; Vincent, P.; Journet, C.; Binh, V. T. Phys. Rev. Lett. 2002, 89, 276103.

(33) Chen, C.; Rosenblatt, S.; Bolotin, K. I.; Kalb, W.; Kim, P.; Kymissis, I.; Stormer, H. L.; Heinz, T. F.; Hone, J. Nat. Nanotechnol. 2009, 4, 861.

(34) Huttel, A. K.; Steele, G. A.; Witkamp, B.; Poot, M.; Kouwenhoven, L. P.; van der Zant, H. S. J. Nano Lett. 2009, 9, 2547.

(35) Gouttenoire, V.; Barois, T.; Perisanu, S.; Leclercq, J. L.; Purcell, S. T.; Vincent, P.; Ayari, A. Small 2010, 6, 1060.

(36) Arcizet, O.; Jacques, V.; Siria, A.; Poncharal, P.; Vincent, P.; Seidelin, S. Nat. Phys. 2011, 7, 879.

(37) Moser, J.; Eichler, A.; Guttinger, J.; Dykman, M. I.; Bachtold, A. Nat. Nanotechnol. 2014, 9, 1007.

(38) Stapfner, S.; Ost, L.; Hunger, D.; Reichel, J.; Favero, I.; Weig, E. M. Appl. Phys. Lett. 2013, 102, 151910.

(39) Singh, V.; Bosman, S. J.; Schneider, B. H.; Blanter, Y. M.; Castellanos-Gomez, A.; Steele, G. A. Nat. Nanotechnol. 2014, 9, 820.

(40) Song, X.; Oksanen, M.; Li, J.; Hakonen, P. J.; Sillanpa, M. A. Phys. Rev. Lett. 2014, 113, 027404.

(41) Weber, P.; Güttinger, J.; Tsioutsios, I.; Chang, D. E.; Bachtold, A. Nano Lett. 2014, 14, 2854.

(42) Schneider, B. H.; Singh, V.; Venstra, W. J.; Meerwaldt, H. B.; Steele, G. A. Nat. Commun. 2014, 5, 5819.

(43) Nigues, A.; Siria, A.; Verlot, P. Nat. Commun. 2015, 6, 8104.

(44) Cole, R. M.; Brawley, G. A.; Adiga, V. P.; De Alba, R.; Parpia, J. M.; Ilic, B.; Craighead, H. G.; Bowen, W. P. Phys. Rev. Appl. 2015, 3, 024004.
(45) Tsioutsios, I.; Tavernarakis, A.; Osmond, J.; Verlot, P.; Bachtold, A. Nano Lett. 2017, 17, 1748.

(46) Güttinger, J.; Noury, A.; Weber, P.; Eriksson, A. M.; Lagoin, C.; Moser, J.; Eichler, C.; Wallraff, A.; Isacsson, A.; Bachtold, A. Nat. Nanotechnol. 2017, 12, 631.

(47) Tavernarakis, A.; Stavrinadis, A.; Nowak, A.; Tsioutsios, I.; Bachtold, A.; Verlot, P. Nat. Commun. 2018, 9, 662.

(48) Huang, S.; Woodson, M.; Smalley, R.; Liu, J. Nano Lett. 2004, 4, 1025.

(49) Jezouin, S.; Parmentier, F. D.; Anthore, A.; Gennser, U.; Cavanna, A.; Jin, Y.; Pierre, F. Science 2013, 342, 601.

(50) Bocquillon, E.; Freulon, V.; Berroir, J.-M.; Degiovanni, P.; Placais, B.; Cavanna, A.; Jin, Y.; Feve, G. Science 2013, 339, 1054.

(51) Jullien, T.; Roulleau, P.; Roche, B.; Cavanna, A.; Jin, Y.; Glattli,

D. C. Nature 2014, 514, 603.

(52) Dong, Q.; Liang, Y. X.; Ferry, D.; Cavanna, A.; Gennser, U.; Couraud, L.; Jin, Y. Appl. Phys. Lett. 2014, 105, 013504.

(53) Song, X.; Oksanen, M.; Sillanpa, M. A.; Craighead, H. G.; Parpia, J. M.; Hakonen, P. J. Nano Lett. 2012, 12, 198.

(54) Weber, P.; Guttinger, J.; Noury, A.; Vergara-Cruz, J.; Bachtold, A. Nat. Commun. 2016, 7, 12496.

(55) Teufel, J. D.; Donner, T.; Castellanos-Beltran, M. A.; Harlow, J. W.; Lehnert, K. W. Nat. Nanotechnol. 2009, 4, 820.

(56) Heritier, M.; Eichler, A.; Pan, Y.; Grob, U.; Shorubalko, I.; Krass, M. D.; Tao, Y.; Degen, C. L. Nano Lett. 2018, 18, 1814.

(57) Reinhardt, C.; Muller, T.; Bourassa, A.; Sankey, J. C. Phys. Rev. $X$ 2016, 6, 021001.

(58) Nichol, J. M.; Hemesath, E. R.; Lauhon, L. J.; Budakian, R. Phys. Rev. B: Condens. Matter Mater. Phys. 2012, 85, 054414.

(59) Wang, Y.; Pistolesi, F. Phys. Rev. B: Condens. Matter Mater. Phys. 2017, 95, 035410.

(60) Degen, C. L.; Poggio, M.; Mamin, H. J.; Rettner, C. T.; Rugar, D. Proc. Natl. Acad. Sci. U. S. A. 2009, 106, 1313.

(61) Mamin, H. J.; Poggio, M.; Degen, C. L.; Rugar, D. Nat. Nanotechnol. 2007, 2, 301.

(62) Nichol, J. M.; Naibert, T. R.; Hemesath, E. R.; Lauhon, L. J.; Budakian, R. Phys. Rev. X 2013, 3, 031016.

(63) Armour, A. D.; Blencowe, M. P.; Zhang, Y. Phys. Rev. B: Condens. Matter Mater. Phys. 2004, 69, 125313.

(64) Clerk, A. A.; Bennett, S. New J. Phys. 2005, 7, 238.

(65) Naik, A.; Buu, O.; LaHaye, M. D.; Armour, A. D.; Clerk, A. A.; Blencowe, M. P.; Schwab, K. C. Nature 2006, 443, 193.

(66) Micchi, G.; Avriller, R.; Pistolesi, F. Phys. Rev. Lett. 2015, 115, 206802.

(67) Pistolesi, F.; Labarthe, S. Phys. Rev. B: Condens. Matter Mater. Phys. 2007, 76, 165317.

(68) Weick, G.; von Oppen, F.; Pistolesi, F. Phys. Rev. B: Condens. Matter Mater. Phys. 2011, 83, 035420.

(69) Micchi, G.; Avriller, R.; Pistolesi, F. Phys. Rev. B: Condens. Matter Mater. Phys. 2016, 94, 125417.

(70) Zippilli, S.; Morigi, G.; Bachtold, A. Phys. Rev. Lett. 2009, 102, 096804.

(71) Santandrea, F.; Gorelik, L. Y.; Shekhter, R. I.; Jonson, M. Phys. Rev. Lett. 2011, 106, 186803.

(72) Stadler, P.; Belzig, W.; Rastelli, G. Phys. Rev. Lett. 2014, 113, 047201 .

(73) Arrachea, L.; Bode, N.; von Oppen, F. Phys. Rev. B: Condens. Matter Mater. Phys. 2014, 90, 125450.

(74) Stadler, P.; Belzig, W.; Rastelli, G. Phys. Rev. Lett. 2016, 117, 197202. 